\title{
Références bibliographiques du dossier « Les défis de l'orientation dans le monde »
}

Bernadette Plumelle et Nicole Siebert-Taabni

\section{OpenEdition}

Édition électronique

URL : http://journals.openedition.org/ries/1572

DOI : 10.4000/ries. 1572

ISSN : 2261-4265

Éditeur

Centre international d'études pédagogiques

Édition imprimée

Date de publication : 1 avril 2005

Pagination : 115-125

ISSN : 1254-4590

\section{Référence électronique}

Bernadette Plumelle et Nicole Siebert-Taabni, « Références bibliographiques du dossier « Les défis de l'orientation dans le monde » », Revue internationale d'éducation de Sèvres [En ligne], 38 | avril 2005, mis en ligne le 11 avril 2012, consulté le 19 avril 2019. URL : http://journals.openedition.org/ries/1572 ;

DOI : 10.4000/ries.1572

(c) Tous droits réservés 


\title{
Références bibliographiques
}

\author{
Nicole Siebert-Taabni \\ Bernadette Plumelle
}

Cette bibliographie non exhaustive est établie par le centre de ressources documentaires du CIEP en collaboration avec le centre de ressources de l'École supérieure de l'éducation nationale (ESEN). Elle recense pour l'essentiel des documents sur l'orientation produits durant ces dix dernières années. Les notices sont organisées autour de trois axes. La première partie présente des études transnationales sur l'orientation en Europe et dans les pays de l'OCDE. La deuxième partie porte sur l'orientation scolaire en France: histoire de l'orientation, orientation scolaire et insertion des jeunes, politiques éducatives et préconisations pour l'amélioration de l'orientation scolaire. Enfin, une dernière partie est consacrée à l'analyse sociologique de l'orientation. Ces documents peuvent être consultés au Centre de ressources documentaires du CIEP ou à celui de l'ESEN; un certain nombre sont en ligne sur Internet. Bibliographie arrêtée le 10 février 2005.

\section{L'orientation scolaire et professionnelle : études transnationales}

CHIOUSSE Sylvie, WERQUIN Patrick, CEDEFOP: Centre européen pour le développement de la formation professionnelle: Fondation européenne pour l'amélioration des conditions de vie et de travail, Conseil et orientation professionnelle tout au long de la vie: éléments de synthèse des expériences menées dans l'Union européenne, Office des publications officielles des communautés européennes/Luxembourg, 1998, 84 p. (Panorama-CEDEFOP)

Cette publication synthétise les résultats du projet Eurocounsel, mené depuis 1991 par la Fondation européenne pour l'amélioration des conditions de vie et de travail, sur le rôle de l'orientation et du conseil pour les adultes. Elle reprend également les résultats de plusieurs projets menés successivement par le CEDEFOP sur l'insertion et l'orientation professionnelles des jeunes et des adultes. Les auteurs ont constaté la diversité des profils des professionnels du conseil; ils ont mis l'accent sur la nécessité pour les praticiens de se mettre au courant de l'évolution du marché du travail et des nouvelles politiques, ainsi que de maintenir des contacts avec différents acteurs locaux et experts du développement communautaire.

Conseil supérieur de l'Éducation/Québec, Au collégial: l'orientation au cour de la réussite. Avis du Conseil supérieur de l'Éducation au ministre de l'Éducation, Conseil supérieur de l'éducation/Sainte-Foy/Canada, avril 2002, 124 p.

Le Conseil supérieur de l'Éducation, organisme consultatif autonome du Québec, livre dans le présent avis une réflexion sur le rôle des collèges, structure intermédiaire entre le cycle secondaire et l'université, par rapport à l'orientation. 
CUISINIER Jean-François, MAC CARTY John, LOVEN Anders et al., L'orientation en Europe: des approches différentes pour une question commune 2 avril 2004, DESCO: Direction de l'enseignement scolaire/ Paris, mai 2004, 41 p., (Les rencontres de la DESCO)

Cette conférence-débat a pour objet un enjeu essentiel du système éducatif: l'orientation. La première contribution décrit la manière dont la problématique de l'orientation a été conçue, mise en œuvre et a évolué en France. Elle est suivie de la présentation du dispositif d'orientation en Suède et d'une analyse comparative des systèmes européens.

HIEBERT Bryan dir., BEZANSON Lynne dir., Faire des vagues: orientation professionnelle et politiques publiques. Le symposium international 1999. Documents, actes et stratégies, Fondation canadienne pour l'avancement de la carrière/Ottawa, 2000

En mai 1999, des décideurs et des intervenants provenant de quatorze pays se sont rencontrés à l'occasion d'un symposium mondial. Chaque pays a rédigé une communication sur son expérience de l'avancement de carrière et sur les défis auxquels il était confronté au plan de la pratique et de la politique. Cette publication réunit l'ensemble des communications ainsi qu'une synthèse des débats, les actes du symposium et une déclaration d'intention internationale.

Faire des vagues : établir un lien entre les services d'orientation et les politiques publiques: volume 2, Fondation canadienne pour l'avancement de la carrièrel Ottawa, 2002

En mars 2001, des responsables et des praticiens de services d'orientation de dix-sept pays et des représentants de quatre organisations multinationales se sont réunis pour un deuxième symposium. Le programme reposait sur cinq enjeux principaux: modèles de politique, résultats, analyse des coûts et avantages, rôle de divers secteurs et normes de formation. Cette publication comprend les documents fournis par les pays, les synthèses sur chacun des cinq enjeux et un résumé des actes.

LECLERCQ Jean-Michel, L'orientation scolaire dans l'Union européenne: choix imposés ou éducation des choix? Administration et éducation, mars 1997, $n^{\circ} 1 ;$ p. 113-119

Depuis les années quatre-vingts, un trait marquant des politiques éducatives de l'Union européenne est l'importance accordée à une orientation non plus imposée mais négociée avec les élèves et même construite par eux. Dans cet article, l'auteur analyse brièvement les efforts des différents pays membres pour substituer aux procédures de sélection des modalités plus positives d'orientation.

McCARTHY John, Les qualifications et la formation des conseillers d'orientation. Document préparé pour l'examen des politiques en faveur des services d'information, d'orientation et de conseil à la demande de la Commission européenne et de l'OCDE, OCDE/Paris, novembre 2001, 21 p. téléchargées du site http://www.oecd.org/

Cette note présente une analyse comparative des qualifications et des formations des conseillers d'orientation, afin de faire apparaître les problèmes qui se posent à l'orientation actuellement. 
OCDE: Organisation de coopération et de développement économiques, Orientation professionnelle: nouvelles pistes de réflexion in: Analyse des politiques d'éducation 2003, OCDE/Paris, 2003, 24 p.

Le chapitre expose l'importance que revêt l'orientation professionnelle du point de vue de l'action des pouvoirs publics et décrit certaines dispositions prises par les pays de l'OCDE. Il donne tout d'abord une description de l'orientation professionnelle. Les sections suivantes indiquent les raisons pour lesquelles l'orientation professionnelle joue un rôle central dans les pays de l'OCDE, en aidant à améliorer le fonctionnement du marché du travail et des systèmes éducatifs et en donnant à chaque personne la possibilité d'enrichir son «capital humain» tout au long de sa vie.

OCDE: Organisation de coopération et de développement économiques, Orientation professionnelle et politique publique: comment combler l'écart, OCDE/ Paris, 2004, 189 p.

Cette publication présente les résultats d'un examen des politiques d'orientation professionnelle entrepris à partir du début de l'année 2001 dans quatorze pays de l'OCDE. Elle étudie les services d'orientation dans des contextes très variés: l'enseignement obligatoire, le deuxième cycle secondaire, l'enseignement tertiaire, les services locaux, le service public de l'emploi et l'entreprise et prend en compte les acteurs de l'orientation: administrations, employeurs, syndicats, institutions éducatives, parents, élèves et praticiens de l'orientation.

OCDE: Organisation de coopération et de développement économiques/ Commission européenne, L'orientation professionnelle: guide pratique pour les décideurs, OCDE/Paris, 2004, 82 p.

Cet ouvrage, publié conjointement par l'OCDE et la Commission européenne, traite de questions fondamentales du point de vue de l'efficacité des services d'orientation professionnelle: comment élargir l'accès à l'orientation? Comment améliorer la qualité de l'information et le pilotage stratégique dans ce domaine? Des propositions sont faites pour améliorer l'orientation professionnelle des jeunes et plus particulièrement de ceux qui quittent prématurément l'école; pour améliorer l'orientation des adultes, l'accès aux services d'orientation professionnelle et enfin, pour améliorer les systèmes d'orientation. De nombreux exemples de bonnes pratiques issues des pays de l'OCDE et d'Europe sont fournis.

WATTS A.G., GUICHARD J., PLANT P. et al., Orientation scolaire et professionnelle dans la Communauté européenne, Bureau des publications officielles des Communautés européennes/Luxembourg, 1994.

Cette étude, financée par la Communauté européenne, a examiné l'évolution des services d'orientation professionnelle, en particulier du point de vue de la qualification et de la formation des personnels de ces services. 


\title{
L'orientation scolaire en France
}

\author{
Histoire de l'orientation \\ en France
}

BLANCHARD Serge, SONTAG Jean-Claude, L'évolution des services, des pratiques et des finalités de l'orientation professionnelle et scolaire en France, Formation et territoire, juillet 2004, $n^{\circ}$ 9, p. 27-44

Les auteurs présentent les grandes étapes de l'évolution des services d'orientation. La description des différentes étapes de l'orientation professionnelle et scolaire à l'éducation nationale est suivie de la présentation des dispositifs mis en place pour les jeunes sortis du système scolaire et pour les adultes. En conclusion, les auteurs dégagent les tendances actuelles de l'évolution des conceptions et des pratiques de l'orientation.

BOUMARD Patrick, MIALARET Gaston, Le conseil de classe, PUF/Paris, 1997, $152 p$.

L'auteur, dans une approche ethnographique, analyse le fonctionnement du conseil de classe qui semble résister au temps et aux diverses réformes. Il a malgré tout évolué tout en restant étrangement stable dans l'imaginaire des élèves, des enseignants et des parents. Signe de pouvoir et d'autorité, il oscille actuellement entre sanction, orientation et évaluation. Son influence reste considérable sur le devenir des élèves et constitue un lieu de décision quant à leur orientation professionnelle.

DANVERS Francis, "Mai 68 " ou la désorientation scolaire et universitaire, Cahiers Alfred Binet, décembre 2001, $n^{\circ}$ 669, p. 37-50

Quelles sont les répercussions de la «montée des jeunes» et de leur révolte sur l'orientation scolaire? Cet article analyse l'évolution des politiques d'orientation scolaire en France au $\mathrm{XX}^{\mathrm{e}}$ siècle, et plus particulièrement l'impact de Mai 1968. Les mécanismes de l'orientation sont en effet liés à l'évolution sociale et politique du pays. L'orientation négociée s'est substituée à l'orientation autoritaire, sans pour autant faire progresser le principe de justice.

PROST Antoine, Des professions à l'école: jalons pour une histoire de l'orientation en France in: L'orientation en perspectives: histoire, récits, analyse, Vie sociale, $n^{\circ} 5,1996$, p. 315-328

L'auteur retrace à grands traits l'origine et l'évolution des centres et des conseillers d'orientation depuis la création du pemier service d'orientation en 1910 jusqu'à la création du diplôme d'État de conseiller d'orientation psychologue (décret du 20 mars 1991).

\section{Orientation scolaire et insertion professionnelle des jeunes}

FEUILLADIEU Sylviane, Projets de lycéens: orientation et projets en classe de seconde générale et technologique, Harmattan/Paris, 2001, 231 p. (Logiques sociales)

Cet ouvrage aborde la question de l'orientation scolaire des lycéens. Pour beaucoup d'entre eux cela implique de réfléchir sur leur trajectoire sociale ultérieure. C'est aussi l'un des moyens 
pour le système éducatif de gérer les flux d'élèves et de réguler l'accès aux diverses formations et qualifications en fonction des besoins économiques. La première partie de l'ouvrage rapporte les résultats d'une enquête conduite par les conseillers-psychologues auprès des élèves de seconde. L'auteur évoque ensuite la place et le rôle de l'entretien d'orientation dans ce processus.

\section{GUICHARD Jean, HUTEAU Michel, Psychologie de l'orientation, Dunod/Paris, 2001, 352 p. (Psycho Sup)}

L'ouvrage constitue une synthèse des recherches sur les choix et conduites d'orientation tout au long de la vie. Il présente les grands courants de recherche en psychologie de l'orientation et leurs principaux résultats et se conclut par une présentation de différentes pratiques d'aide à l'orientation. L'ouvrage traite à la fois de l'orientation scolaire et professionnelle des jeunes à l'intérieur du système éducatif et de l'orientation professionnelle des adultes.

GUICHARD Jean coord., Les enjeux de l'orientation: dossier, Éducations, revue de diffusion des savoirs en éducation, $n^{\circ} 11$, février 1997, p. 14-57, bibliogr.

La majorité des articles de ce dossier évoquent les processus d'orientation scolaire et professionnelle des élèves: le contexte social, la place des projets personnels et des contraintes structurelles, le rôle des résultats et appréciations, l'éducation des choix, la conception éducative de l'orientation, la perception de l'orientation par les élèves, la notion de projet et les différentes orientations des filles et des garçons.

\section{GUIGUE-DURNING Michèle, Le point de vue des jeunes sur l'orientation en} milieu scolaire, L'Harmattan/Paris, 2001, 268 p. (Savoir et formation)

Étude sur l'orientation scolaire et professionnelle des élèves au collège: réflexion sur l'éducation à l'orientation au collège, analyse du point de vue de quatre collégiens parisiens sur l'orientation; étude d'itinéraires scolaires et professionnels sur plusieurs années de jeunes en difficultés placés en foyer, entrés en apprentissage et d'élèves allemands évoluant dans un système scolaire à filières.

\section{L'Orientation face aux mutations du travail ( $\left.L^{\prime}\right)$ : actes du colloque L'orienta- tion tout au long de la vie, La Villette 26-28 septembre 1996, Syros/Paris, Cité des sciences et de l'industrie/Paris, 1997, 263 p. \\ Cet ouvrage est issu du colloque organisé dans le Cadre de l'année européenne de l'éducation et de la formation tout au long de la vie. Il met en perspective des situations concrètes qu'ont à résoudre quotidiennement les professionnels de l'éducation, de la formation et des ressources humaines dans un contexte économique et professionnel en mutation.}

\section{SENECAT Jacques, DENQUIN Robert, MOISSON Jeanine, L'orientation, Cahiers de l'Éducation, avril 1999, $n^{\circ} 3$, p. 2-19}

Selon la loi du 10 juillet 1989 relative à l'Éducation, l'orientation doit constituer un «projet personnel» dans la vie de l'élève et de l'étudiant. Ce dossier propose un panorama des aspects de la question (approche historique, pédagogique, administrative et technique) à travers les différents niveaux du système éducatif (collège, lycée, LEP, université).

\section{VIOLET Daniel, L'Orientation scolaire et professionnelle: vers une démarche} initiatique, Cahiers Alfred Binet, décembre 2001, $n^{\circ}$ 669, p. 83-96

La loi d'orientation de 1989 veut faire de chaque élève l'acteur de son projet personnel. Cet article propose l'étude de quelques pratiques d'application de ce principe et montre que la réalité ne correspond pas toujours à cette intention. Une part d'imaginaire, de symbolique et de mystère doit être préservée dans le processus d'orientation scolaire et professionnelle, qui n’obéit pas à des principes d'efficacité stricts. 


\title{
Les politiques d'orientation scolaire en France
}

\author{
BENHAÏM Jeanne, Les politiques en faveur de l'éducation à l'orientation en \\ collège et lycée général et technologique, Note d'information, $n^{\circ}$ 03-18, mars \\ 2003, 6 p. \\ Les directeurs des centres d'information et d'orientation (CIO) constatent la mise en ouvre \\ effective de l'éducation à l'orientation dans seulement $55 \%$ des collèges et $37 \%$ des lycées en \\ raison de la difficulté de concevoir une politique globale au sein des établissements.
}

BELLOUBET-FRIER Nicole, 30 propositions sur l'avenir du lycée, Ministère de l'éducation nationale et de la recherche/Paris, mars 2002, 76 p. téléchargées du site www.education.gouv.frl

L'auteur centre sa réflexion sur les points suivants: analyse des raisons de la stagnation du taux d'accès d'une génération de lycéens au baccalauréat et volonté d'accroître la proportion de bacheliers; propositions visant à recentrer les missions de l'enseignement secondaire aussi bien dans la voie générale que technologique ou professionnelle; redéfinition des processus d'orientation au collège, de la liaison collège/lycée et lycée/études supérieures.

BRUNEL Olivier coord., 99 questions sur... l'éducation à l'orientation, CRDP Languedoc-Roussillon, 2001, n.p.

À partir de leurs expériences et de leur recherche, les auteurs proposent, en 99 fiches, un panorama de l'éducation à l'orientation qui s'organise autour de plusieurs thèmes: origine, organisation et pilotage dans l'établissement, mise en œuvre pédagogique, acteurs. Un dernier chapitre définit les enjeux et les effets de cette éducation à l'orientation.

Commission du débat national sur l'avenir de l'École/Paris, Les Français et leur École: le miroir du débat, Dunod/Paris, 2004, 575 p., annexes

Cet ouvrage présente l'éventail des opinions qui se sont exprimées lors du débat national sur l'école de septembre 2003 à mars 2004. Dans le chapitre "Faire réussir les élèves», une partie est consacrée à l'orientation (pp. 100-108). Le point de vue des jeunes sur leur orientation est rappelé et des préconisations sont proposées du point de vue des structures et des acteurs (entreprises, conseillers d'orientation-psychologues et enseignants).

\section{CYTERMANN Jean-Richard, Parcours dans l'enseignement secondaire et terri-} toires, Éducation \& Formations, janvier 2002, nº 062, p. 5-105.

Les notions importantes sur les parcours de formation des élèves dans l'enseignement secondaire ont été dégagées lors du séminaire de la direction de la programmation et du développement (DPD) du ministère de l'Éducation en juin 2001. Les trajectoires des élèves sont clairement visualisées comme la part respective de l'enseignement général et de l'enseignement professionnel, l'efficacité scolaire des régions, les flux d'élèves que s'échangent les différents appareils de formation publics et privés.

DENQUIN Robert, ROYNETTE Alain, SERE Alain, FRANCE, Inspection générale de l'éducation nationale, L'orientation vers le lycée professionnel. La scolarisation au lycée professionnel. Rapport à Monsieur le Ministre de l'Éducation nationale, Inspection générale de l'éducation nationale/Paris, janvier 2002, 61 p. téléchargée du site: http:/llesrapports.ladocumentationfrancaise.fr 
Après avoir étudié les pratiques d'orientation vers les lycées professionnels, le présent rapport estime nécessaire de faire évoluer l'enseignement professionnel. Il définit la mission d'orientation de l'Éducation nationale, analyse le passage de l'école vers le monde du travail, constate l'évolution des effectifs, les inégalités face à l'information et à l'orientation. Il émet des propositions destinées à soutenir l'installation et l'orientation vers la voie professionnelle dans une logique de qualité. Le rapport est consultable sur Internet.

GROSBRAS Francine coord., L'éducation à l'orientation au collège, ONISEP/ Paris, Hachette Éducation/Paris, CNDP/Paris, 1998, 158 p., bibliogr., (Ressources formation. Enjeux du système éducatif)

L'éducation à l'orientation est devenue l'une des missions premières de l'École et fait l'objet d'un consensus repris dans les pratiques des établissements et les textes officiels. Elle nécessite une «pédagogie de l'autodétermination» et la connaissance du développement de l'adolescent ainsi qu'une étude du monde du travail et du contexte économique et social.

GUICHARD Jean éd., GUILLON Vincent éd., LOWIT Valérie éd., Éduquer en orientation: enjeux et perspectives. Actes du colloque international INETOPCNAM Paris 24-26 mai 2000, L'Orientation scolaire et professionnelle (L'), Vol. 30, $n^{\circ}$ hors-série, juillet 2001, 606 p.

L'éducation en orientation s'est fortement développée au cours des vingt-cinq dernières années ce qui est en partie dû à la scolarisation de masse. Ce hors série, proposant les actes du colloque consacré à la problématique de l'orientation, tente de répondre à ce questionnement. Les conférences, communications, ateliers, symposiums, table ronde relatés montrent que l'éducation en orientation est devenue «une évidence intellectuelle mais pas encore une pratique totalement généralisée».

HCéé: Haut Conseil de l'évaluation de l'école, L'évaluation de l'orientation à la fin du collège et au lycée, HCéé/Paris, mars 2004, $n^{\circ} 12,4$ p. téléchargées du site: http://cisad.adc.education.fr/hcee (Avis du HCéé)

Le Haut Conseil constate que, dans une large mesure, l'orientation concentre les critiques sur le système éducatif. La clarification des objectifs et des enjeux de l'orientation est l'étape indispensable pour préciser les missions des instances et des personnels de l'orientation.

HENOQUE Maryse, LEGRAND André, L'évaluation de l'orientation à la fin du collège et au lycée. Rêves et réalités de l'orientation. Rapport établi à la demande du Haut conseil de l'évaluation de l'école, Haut conseil de l'évaluation de l'école/Paris, $n^{\circ}$ 12, mars 2004, 93 p.

Ce rapport retrace les politiques publiques concernant l'orientation, les divergences entre objectifs et résultats et les lacunes qui subsistent sur la connaissance des processus d'orientation. Les auteurs soulignent la nécessité de gérer la contradiction entre les deux objectifs indissociables de l'orientation: "gestion des flux», compte tenu des évolutions des besoins économiques et sociaux, et «construction progressive d'un projet individuel de l'élève». Enfin, les auteurs examinent la situation de l'orientation dans d'autres pays (pays de l'OCDE) afin d'observer les difficultés rencontrées et les solutions préconisées.

\section{MELLON Patrick, L'orientation au collège: évolutions depuis 1975, enjeux et} perspectives, Administration et éducation, janvier 2002, $n^{\circ} 093$, p. 33-45

La question de l'orientation au collège a profondément évolué. La délimitation entre formation professionnelle et filière générale n'est plus faite au seuil de la quatrième mais à celui de la seconde. Désormais, l'éducation à l'orientation est un véritable enjeu pour le collège et le rôle du conseiller d'orientation-psychologue s'en trouve modifié. 
Ministère de l'éducation nationale/Paris, L'orientation vers le lycée professionnel, Ministère de l'éducation nationale, janvier 2002, n²002-003, 51 p. téléchargées du site siteftp://trf.education.gouv.frl

Les rapporteurs se sont attachés à appréhender, à donner à percevoir les aspects du fonctionnement de l'orientation ainsi qu'à cerner les facteurs intervenant dans la construction et l'évolution de la représentation de l'enseignement professionnel chez les adolescents. Ce rapport, bâti en trois parties, détermine les problématiques liées à l'enseignement professionnel, décrit l'évolution des effectifs et la perception de l'orientation par l'ensemble des acteurs et établit un certain nombre de propositions.

Ministère de la jeunesse, de l'éducation nationale et de la recherche/Paris, Propositions pour améliorer le processus d'orientation et les procédures d'affectation au lycée professionnel, Ministère de l'éducation nationale, décembre 2003, $n^{\circ}$ 2003-99, 28 p. téléchargées $d u$ siteftp://trf.education.gouv.fr/pub/edutel/syst/igen/rapports/orient_aff.pdf

L'amélioration de l'orientation et de l'affectation au lycée professionnel constitue un enjeu fort pour le système scolaire. Après un état des lieux portant sur l'éducation à l'orientation, les logiques divergentes entre orientation et affectation et un premier diagnostic, les auteurs définissent un parcours-type de la classe de sixième à la classe de troisième et les principes de l'éducation à l'orientation. Le rapport se termine par des propositions d'actions à différents niveaux (académies, bassins, collèges).

PERETTI Claudine dir., Les processus d'orientation sont-ils équitables et efficaces? In Dix-huit questions sur le système éducatif, La Documentation française/Paris, 2004, p., 57-75

Il existe des paliers d'orientation particulièrement importants dans le système scolaire français: en fin de classe de troisième, en fin de seconde pour les filières générale et technologique et à l'entrée de l'enseignement supérieur. Les élèves sont-ils orientés en fonction de leur valeur scolaire? L'étude produite ici fait apparaître de très grandes différences selon l'origine sociale, le sexe et l'académie d'appartenance.

\section{REBAUD Claude, VIGNA Régine, BOULU Jean-Claude et al., Pour une politique} d'orientation en établissement, Les cahiers d'Éducation \& Devenir, septembre 2004, $n^{\circ} 3,81 p$.

Ce cahier, réalisé par différents acteurs du système éducatif (chefs d'établissement, conseillers d'orientation-psychologues, conseillers principaux d'éducation), propose trois types de textes: articles de fond, témoignages et fiches techniques. Il attache une importance particulière au conseil de classe, considéré comme un moment clé de l'orientation et aborde la construction du projet d'orientation dans l'établissement: lieux, acteurs, dispositifs.

\section{SOLAUX Georges, L'éducation à l'orientation : les directives ministérielles et l'état de la recherche, L'orientation scolaire et professionnelle, 1999, $n^{\circ} 28$}

L'auteur analyse les concepts, méthodes et outils proposés par le ministère de l'Éducation nationale (MEN) en vue de développer l'éducation à l'orientation et détermine le modèle théorique que le MEN a construit de l'élève placé devant des alternatives de poursuite d'études. Il étudie également les correspondances ou les rencontres entre les connaissances produites par le champ scientifique et les conceptions actuelles de l'orientation au collège développées par l'Éducation nationale. 


\section{Études sociologiques sur l'orientation}

BERTHELOT Jean-Michel, École, orientation, société, PUF/Paris, 1993. 192 p. (pédagogie d'aujourd'hui)

L'auteur propose une nouvelle approche sociologique de la question de l'orientation, en prenant notamment appui sur les résultats d'une étude de suivi menée auprès d'un groupe de lycéens durant cinq années et qui met en évidence la complexité des rapports entre le système scolaire et la société, la surdétermination des situations d'orientation et l'irréductibilité des individus concernés.

BULLE Nathalie, Simulation des choix de filières scolaires: application à l'orientation des élèves dans le second cycle du secondaire depuis le début du siècle en France, Revue française de sociologie, décembre 1996, vol. XXXVII, $n^{\circ} 4$; p. 567-604, bibliogr.

\section{CAILLE Jean-Paul, LEMAIRE Sylvie, VROLANT Marie-Claude, Filles et garçons} face à l'orientation, Note d'information, avril 2002, $n^{\circ} 02.12$, p. 1-6

Portant sur les choix d'orientations des collégiens et lycéens, cette étude montre que le choix des élèves entre enseignement général et professionnel à la fin du collège dépend de leur niveau scolaire. En revanche, les choix entre les différents BEP et les différentes filières de l'enseignement général et technologique apparaissent sexués: les garçons dominent dans les filières scientifiques et dans le secteur secondaire.

CHAZAL Sébastien, GUIMOND Serge, La théorie de la dominance sociale et les choix d'orientation scolaire et de rôles sociaux des filles et des garçons, Orientation scolaire et professionnelle, décembre 2003, vol. 32/04, p. 595-616

Cette recherche a comme objectif d'examiner le rôle de la dominance sociale dans l'explication des choix d'orientation scolaire et de rôles professionnels des élèves en classe de seconde. Elle confirme la pertinence de cette théorie pour la compréhension des facteurs qui motivent les choix d'orientation contrastés des filles et des garçons.

DURU-BELLAT Marie, VAN ZANTEN Agnès, Sociologie de l'école, Armand Colin/Paris, 2002, 252 p. (collection U)

Cet ouvrage présente une synthèse des constructions théoriques et des résultats empiriques aujourd'hui disponibles sur l'école. On y trouve une présentation critique des contraintes qui pèsent sur le système scolaire français ainsi que des analyses détaillées sur de nombreux thèmes dont celui de l'orientation.

DURU-BELLAT Marie, Actualité et nouveaux développements de la question de la reproduction des inégalités sociales par l'école, Orientation scolaire et professionnelle, décembre 2003, vol. 32/04, p. 571-594

À partir de travaux récents mis en perspective, sont abordés les différents mécanismes qui concourent à la production des inégalités scolaires en France: les mécanismes de nature individuelle et ceux de type contextuel et les stratégies des familles face aux inégalités de l'offre. 
DURU-BELLAT Marie, JAROUSSE Jean-Pierre, SOLAUX Georges, S'orienter et élaborer un projet au sein d'un système hiérarchisé, une injonction paradoxale? L'exemple du choix de la série et de l'enseignement de spécialité en classe terminale, L'orientation scolaire et professionnelle, 1997, $n^{\circ} 4$, pp. 459-482

La scolarité en lycée est marquée par des choix successifs d'options et de filières, de la classe de troisième à la classe terminale. Cet article analyse sur une base empirique la détermination progressive de ces choix, c'est-à-dire leur genèse temporelle et les facteurs scolaires et sociaux associés. De fait, ces choix scolaires semblent moins obéir à une logique pédagogique qu'à un souci d'optimiser sa carrière scolaire.

DURU-BELLAT Marie, MINGAT Alain, Orientation scolaire et mécanismes institutionnels: mesures des inégalités sociales en France depuis trente ans et comparaisons internationales, PULIM: Presses de l'université de Limoges et $d u$ Limousin, 1992, 73 p. (Didactica)

Les travaux sur l'orientation scolaire menés par Marie Duru-Bellat et Alain Mingat ont montré l'importance des facteurs institutionnels dans la "genèse des carrières scolaires des élèves ». Les auteurs se sont attachés, ici, à analyser les processus d'orientation dans plusieurs pays. L'étude porte sur cinq pays européens avec trois monographies sur l'Italie, le RoyaumeUni et la Suisse et propose une analyse rétrospective de la situation française sur les vingt dernières années.

DURU-BELLAT Marie, Le fonctionnement de l'orientation: Genèse des inégalités sociales à l'école, Delachaux et Niestlé, 1988, 199 p., ill., Bibliogr. (Actualités pédagogiques et psychologiques)

Cet ouvrage présente à la fois les résultats de recherche et une synthèse des travaux existants concernant les mécanismes de l'orientation scolaire. Il peut aussi constituer une initiation à la sociologie de la réussite scolaire.

\section{LE BASTARD-LANDRIER Séverine, Les déterminants contextuels de l'orienta-} tion scolaire en classe de seconde, Les Sciences de l'éducation pour l'ère nouvelle, 2004, vol. $37, n^{\circ} 2$; p. 59-81

Les résultats de cette recherche soulignent l'aspect inégalitaire du processus d'orientation en classe de seconde selon l'établissement fréquenté par les élèves, et notamment selon la composition sociale de son public. Ainsi, l'orientation apparaît comme un processus interne qui relève de la politique de l'établissement scolaire et qui consiste en partie à gérer la composition sociale du public en répartissant les élèves de façon plus ou moins égalitaire dans les différentes séries.

MERLE Pierre, Sociologie de l'évaluation scolaire, PUF/Paris, 1998, 127 p., bibliogr., (Que sais-je?)

Justice de la notation, pertinence des décisions d'orientation et de redoublement, liens entre dispositifs pédagogiques (ZEP, préscolarisation...) et performances scolaires des élèves, telles sont les questions abordées dans cet ouvrage.

L'Orientation: différence de comportement entre filles et garçons, L'Enseignement technique, septembre 2002, $n^{\circ} 195$, p. 13-14

Grâce à cette analyse statistique, on démontre que les filles optent moins souvent que les garçons pour une première scientifique. En première technologique comme en BEP, les sections industrielles restent le domaine réservé des garçons alors que les filles rejoignent en majorité les sections tertiaires. Dans l'enseignement supérieur les filles se dirigent plus souvent vers des études longues et les garçons vers une filière sélective. 
RINGER Fritz, GEAY Bertrand, MILLET Mathias et al., Les contradictions de la démocratisation scolaire, Actes de la recherche en sciences sociales, 2003, $n^{\circ} 149$; $95 \mathrm{p}$.

Ce numéro étudie les transformations du système d'enseignement français face à la massification scolaire et aux restructurations qui l'ont accompagnée. Il interroge également les multiples dispositifs de gestion des flux scolaires: dispositifs institutionnels pour élèves en difficulté, hiérarchisation des filières de l'enseignement secondaire, modalités différentes d'accès à l'enseignement supérieur.

ROUX Sébastien, DAVAILLON Alice, Le processus d'orientation en fin de troisième: observation des comportements des acteurs et analyse des causalités, Éducation \& Formations, juillet 2001, $n^{\circ} 060$, p. 41-53

La présente étude, qui se propose d'analyser les différentes phases du processus d'orientation, est particulièrement centrée sur la recherche des facteurs de disparité au cours de ce processus. Il s'agit par exemple des choix d'orientation des familles en fonction de la valeur scolaire de l'élève, de l'ambition de la famille selon le milieu auquel elle appartient, de l'impact du sexe de l'élève et de la structure familiale dans les choix d'orientation.

VOUILLOT Françoise, Enjeu et mise en jeu de l'identité sexuée dans les conduites d'orientation In: Quelle mixité pour l'école, SCEREN/CNDP, Albin Michel/Paris, 2004, p. 47-55. (Les débats du CNP)

Cet ouvrage fait suite au colloque «L'enseignement est-il mixte?» organisé par le Conseil national des programmes (CNP) en avril 2004. Françoise Vouillot s'interroge sur la manière dont la mixité est vécue dans l'enseignement et sur les causes de la persistante inégalité entre les sexes. 
